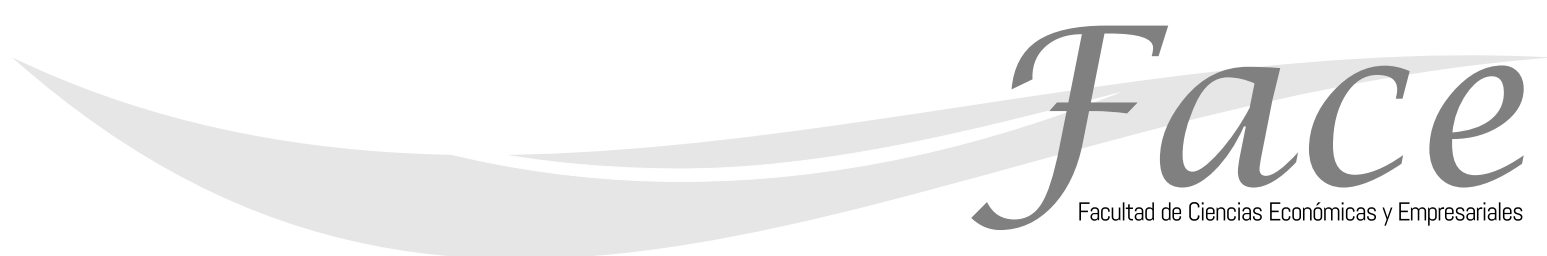

ISSN: Impreso: 1794-9920

ISSN Electrónico: 2500-9338

Volumen 17 - №2

Año 2017

Págs 176 - 184

\title{
EFECTOS DEL NUEVO SISTEMA GENERAL DE REGALÍAS (SGR) SOBRE EL DESEMPEÑO FISCAL DEL DEPARTAMENTO DE CASANARE
}

Fecha de recepción: 29 Febrero de 2017

Fecha de aprobación: 27 Abril de 2017

José Andrés Alvarez Sierra*

Enlace ORCID: http://orcid.org/0000-0002-1408-1767

\begin{abstract}
Resumen:
En este documento se evalúan los efectos de la implementación del Sistema General de Regalías (SGR) sobre el desempeño fiscal del Departamento, entre los recursos más importantes con que cuenta para consolidar el desarrollo y competitividad territorial de Casanare tenemos las regalías, que venían siendo ejecutadas con inequidad, ineficiencia y corrupción. Por esto, el Gobierno nacional realizó la modificación constitucional conocida como Acto Legislativo 005 de 2011.

A partir de este acto legislativo se ha venido reglamentando el Sistema General de Regalías (SGR) de manera radical y en especial su distribución, así mismo la forma de cómo acceder y ejecutar dichos recursos. Debido a estos importantes cambios, en el presente documento se realiza un análisis del comportamiento histórico del anterior régimen y el nuevo Sistema General de Regalías (SGR) y el efecto sobre el desempeño fiscal del Departamento de Casanare.
\end{abstract}

Palabras Claves: DNP: Departamento Nacional de Planeación; SGR: Sistema General de Regalías; Desempeño Fiscal del Departamento; Indicador Fiscal.

\footnotetext{
* Matemático con Énfasis en Estadística, Especialista en Estadística Aplicada, Especialista en Gerencia de Proyectos, Administrador de Empresas Universidad de Pamplona; Asesor Despacho Gobernador de Casanare y Catedrático Universidad Unisangil, Sede Yopal; con experiencia en $f$ inanzas en el área de Salud y administración de Entes Territoriales Departamentales. Email: joseandres114@hotmail.com
} 


\title{
EFFECTS OF THE NEW GENERAL SYSTEM OF ROYALTIES (SGR) ON THE FISCAL PERFORMANCE OF THE DEPARTMENT OF CASANARE
}

\begin{abstract}
:
In this document, the effects of implementation of General System of Royalties are evaluated about the fiscal performance, between the most important resources to consolidate the development and territorial competitiveness, Casanare have royalties that were poorly executed with inequity, inefficiency and corruption. Because of this, National government made modification of the Constitution like The Acto Legislativo 005 de 2011.

From this legislative act has been regulated the General System of Royalties (GSR) radically and mainly the way to access and execute these resources. Due to these important changes, this document analysis will make a comparative analysis between the previous and the new system and the effect on the fiscal performance of Casanare.
\end{abstract}

Keywords: National Department of Planning (NDP); General System of Royalties (GSR); fiscal performance of the Department; fiscal indicator.

\section{EFEITOS DO NOVO SISTEMA GERAL DE ROYALTIES (SGR) SOBRE O DESEMPENHO FISCAL DO DEPARTAMENTO DE CASANARE}

\section{Resumo:}

Neste documento, os efeitos da implementação do Sistema Geral de Royalties são avaliados quanto ao desempenho fiscal, entre os recursos mais importantes para consolidar o desenvolvimento e a competitividade territorial, Casanare possui royalties mal executados com iniqüidade, ineficiência e corrupção. Devido a isso, o governo nacional fez modificação da Constituição como 0 Acto Legislativo 005 de 2011.

A partir deste ato legislativo foi regulamentado o Sistema Geral de Royalties (GSR) de forma radical e principalmente a forma de acessar e executar esses recursos. Devido a essas importantes mudanças, esta análise documental fará uma análise comparativa entre o sistema anterior e o novo e o efeito sobre o desempenho fiscal de Casanare.

Palavras chave: National Department of Planning (NDP); General System of Royalties (GSR); fiscal performance of the Department; fiscal indicator. 


\section{INTRODUCCIÓN:}

El objetivo del presente documento es, determinar los efectos del nuevo Sistema General de Regalías (SGR) sobre el Desempeño Fiscal del Departamento de Casanare, que sirva de insumo para la formulación de políticas públicas y estrategias que contribuyan al fortalecimiento del sector y a la atenuación de los impactos por la reducción de los ingresos.

Así mismo analizar el comportamiento de los ingresos y gastos del departamento, con el antiguo sistema de regalías y el nuevo SGR, estableciendo el efecto que ha tenido sobre las finanzas del departamento, adicionalmente establecer la relación del nuevo sistema sobre el desempeño fiscal del Departamento.

Igualmente, y ante el difícil panorama de la economía regional por la pronunciada recesión del sector petrolero, se hace necesario plantear alternativas de solución, que nos permitan avanzar, integrando el sector gremial, los municipios, el departamento y la sociedad, en aras de fomentar un entorno propicio para incentivar la inversión privada en proyectos o negocios que nos ayuden a dinamizar y reactivar la economía Departamental.

\section{MARCO TEÓRICO:}

El Departamento de Casanare hace parte de la denominada región de la Orinoquía junto con los departamentos de Arauca, Guainía, Guaviare, Meta, Vichada y Vaupés. Casanare es un territorio relativamente joven ya que fue erigido a la categoría de departamento en el año 1991 con la nueva Constitución Política.

La economía de Casanare se basa principalmente en la producción ganadera, agrícola y en la explotación petrolera (Repositorio Universidad del Rosario). El departamento de Casanare es conocido en el país por el tema petrolero; las bonanzas que la región ha tenido en la explotación de hidrocarburos han significado un factor de cambio importante, lo que ha motivado problemas entre los gobiernos central, departamental y municipal; dificultando las relaciones entre los sectores público, privado y la sociedad civil, por lo tanto los proyectos de inversión social se han limitado a los manejos del estado como eje de desarrollo económico.

Casanare se sustentó durante muchos años en la tradicional ganadería extensiva, con baja inversión en tecnología y empleo; complementada con explotaciones agrícolas de pequeña escala e incipientes actividades de comercio y servicios. A partir de 1995 inició el auge del subsector minero, siendo relegados a un segundo plano los demás renglones; es así como, gracias a la producción intensiva de petróleo, el PIB de este departamento mostró en la década de los noventa una tasa promedio anual de crecimiento de $11,8 \%$; resultado notablemente superior al alcanzado por el agregado nacional, que se ubicó en 2,7\%. (Banco de la República, 2014).

El Banco de la República en el análisis de la composición de la economía del Departamento de Casanare, concluye que el valor agregado por grandes sectores en Casanare para el período 1991-2013 presentó una distribución marcadamente acentuada hacia el primario, en donde se concentró el grueso del valor de la producción, muy distante del secundario y el terciario. Indudablemente, el peso en este resultado lo asumió el sector minero, como resultado de la la explotación de petróleo crudo y gas natural. Específicamente, entre 2001 y 2013 la participación promedio anual del sector primario en el PIB departamental superó el $75,0 \%$, en tanto que el siguiente en importancia, pero muy alejado, fue el terciario, que para este lapso sobrepasó el 14\%, mientras que el aporte del secundario fue todavía más bajo, con una media anual de 7,7\% (Banco de la República, 2014).

El Informe de Coyuntura Económica Regional (ICER), publicado en Octubre de 2016 para el Departamento de Casanare\%, por el DANE; indican que "De acuerdo a las ramas de actividad económica, la mayor participación de Casanare en 2014p fue en extracción de petróleo crudo y de gas natural; actividades de servicios relacionadas con la extracción de petróleo y de gas, excepto las actividades de prospección; extracción de minerales de uranio y de torio con $68,1 \%$; seguido de cultivo de otros productos agrícolas $(3,5 \%)$, producción pecuaria y caza incluyendo las actividades veterinarias $(3,4 \%)$, administración pública y defensa; seguridad social de afiliación obligatoria $(2,8 \%$ ) y construcción de edificaciones completas y de partes de edificaciones; acondicionamiento de edificaciones $(2,7 \%)$. Estas ramas tuvieron variaciones anuales de 4,3\%, 3,6\%, $1,3 \%, 3,9 \%$ y $411,8 \%$, respectivamente"(DANE, 2016).

Uno de los principales problemas que está atravesando el Departamento de Casanare es la escases de la producción petrolera y la disminución del valor del barril de crudo, así como los ajustes a los ingresos de las entidades territoriales por cuenta del SGR creado por el Acto Legislativo 05 del 18 de Julio de 2011 "Por la cual se constituye el Sistema General de Regalías, se modifican los artículos 360 y 361 de la Constitución política y se dictan otras disposiciones sobre 
ISSN: 1794-9920 - ISSN Electrónico: 2500-9338

Volumen 17- Número 2, Año 2017- Págs: 176 - 184.

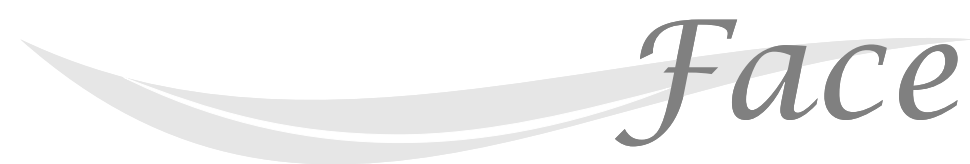

el Régimen de Regalías y Compensaciones" (CONGRESO DE COLOMBIA, 2011). Que ha generado un proceso de reestructuración a las finanzas del Departamento y mientras esto sucede, el dinamismo y crecimiento económico entraron en un estancamiento.

Con la modificación a la Constitución Política de Colombia y a partir de la entrada en vigencia del nuevo Sistema General de Regalías (SGR) creado por el Acto Legislativo 05 del 18 de Julio de 2011 "Por la cual se constituye el Sistema General de Regalías, se modifican los artículos 360 y 361 de la Constitución política y se dictan otras disposiciones sobre el Régimen de Regalías y Compensaciones", el concepto y distribución de regalías en Colombia cambio y el nuevo texto del artículo 360 de la Constitución política de Colombia quedó así: "La explotación de un recurso natural no renovable causará, a favor del Estado, una contraprestación económica a título de regalía, sin perjuicio de cualquier otro derecho o compensación que se pacte. La ley determinará las condiciones para la explotación de los recursos naturales no renovables. Mediante otra ley, a iniciativa del Gobierno, la ley determinará la distribución, objetivos, fines, administración, ejecución, control, el uso eficiente y la destinación de los ingresos provenientes de la explotación de los recursos naturales no renovables precisando las condiciones de participación de sus beneficiarios. Este conjunto de ingresos, asignaciones, órganos, procedimientos y regulaciones constituye el Sistema General de Regalías"(ACTO LEGISLATIVO 05 DE 2011).

A partir de este momento la participación de las entidades territoriales en las regalías, quedo centralizada y a merced del Gobierno Nacional, en adelante sería el Estado como titular de las regalías quien transfiere, a título de participación, un porcentaje de las regalías. Con ello busca beneficiar a las entidades descentralizadas territorialmente, para que fomenten el desarrollo.

Teniendo en cuenta los efectos que este nuevo sistema de asignación de regalías, pudo traer para el Departamento de Casanare, se plantea esta propuesta de investigación que pretende evaluar cómo ha sido el efecto del nuevo sistema general de regalías sobre el desempeño fiscal del Departamento de Casanare, se trata de establecer, cómo ha sido el comportamiento de los ingresos del Departamento antes y después del nuevo SGR?; cuál ha sido el comportamiento del gasto de la entidad territorial desde la fecha del nuevo SGR?; si existe o no una relación entre los ingresos por asignaciones del SGR y el desempeño fiscal del Departamento de Casanare?

Metodología
El diseño de estudio para el análisis del impacto del nuevo SGR sobre el desempeño fiscal del Departamento de Casanare, es retrospectivo y descriptivo.

Para llevar a cabo esta investigación, las fuentes de información más importantes fueron las ejecuciones presupuestales desde el año 2000 al año 2016, que fueron suministradas por la Secretaría de Hacienda Departamental y las publicadas por el DNP en su sitio Web en lo relacionado con la información del plan de Recursos del SGR. Adicionalmente se utilizó la información oficial de los resultados del desempeño fiscal de los departamentos (DNP, 2018).

Para la estimación de los recursos asignados con cargo al SGR para el año 2012; bienios: 2013 - 2014, 2015 - 2016, 2017 - 2018, se tuvieron en cuenta las cifras de los presupuestos publicadas por el Ministerio de Hacienda en la página oficial, así:

$\checkmark$ Año 2012: Presupuesto del Sistema General de Regalías Decreto 4950 de 2011 y apropiación ajustada Decreto 1399 de 2012.

$\checkmark$ Bienio 2013 - 2014: Presupuesto del Sistema General de Regalías Ley 1606 de 2012

$\checkmark$ Bienio 2015-2016: Presupuesto del Sistema General de Regalías Ley 1744 de 2014.

$\checkmark$ Bienio 2015 - 2016: Presupuesto del Sistema General de Regalías Decreto 2190 de 2016.

A continuación se presentan los resultados del desempeño fiscal del departamento de Casanare, de conformidad con lo establecido en la Ley 617 de 2000 y según los criterios de evaluación establecidos por la Dirección de Desarrollo Territorial Sostenible del Departamento Nacional de Planeación, publicados y disponibles desde el año 2000 hasta el año 2016.

Los rangos clasifican a las entidades territoriales de acuerdo con los resultados ubicándolas entre las más débiles o las más avanzadas de acuerdo con el lugar que ocupen en la evaluación fiscal, esos rangos se clasificaron en:

Tabla 1. Rangos de Medición del componente de desempeño fiscal

\begin{tabular}{ll}
\hline NIVELES DE DESEMPEÑO FISCAL & RANGOS DEMEDICIÓN \\
\hline Solvente & $\geq 80$ \\
Sostenible & $\geq 70 y<80$ \\
Vulnerable & $\geq 60 y<70$ \\
Riesgo & $\geq 40 y<60$ \\
Deterioro & $<40$ \\
\hline
\end{tabular}

Fuente: Desempeño Fiscal de los Departamentos: https://www.dnp.gov.co/programas/desarrollo-territorial/EstudiosTerritoriales/Indicadores-y-Mediciones/Paginas/desempeno-fiscal.aspx 
La metodología de evaluación fiscal aplicada en esta medición, es la realizada por la Dirección de Desarrollo Territorial Sostenible del Departamento Nacional de Planeación.

Los datos que se obtuvieron en el trabajo de campo, fueron revisados asegurando la calidad y que la fuente de la información tengan un nivel de confiabilidad alto.

De igual manera se analizó mediante la técnica estadística de regresión lineal la relación de dos variables, una dependiente y la otra independiente, la posible relación entre el desempeño fiscal y los ingresos del Departamento por concepto de regalías.

Para el análisis de los ingresos y gastos, conforme a los datos de las ejecuciones presupuestales, la técnica estadística que se aplicó para realizar el presente análisis fue una serie de tiempo, que permite analizar algunos componentes básicos como la tendencia, estacionalidad y aleatoriedad, con el propósito de inferir sobre las variaciones que puedan llegar a encontrarse y sus posibles causas.

La herramienta estadística empleada para el procesamiento de los datos fue el paquete estadístico SPSS versión 22.0

\section{RESULTADOS::}
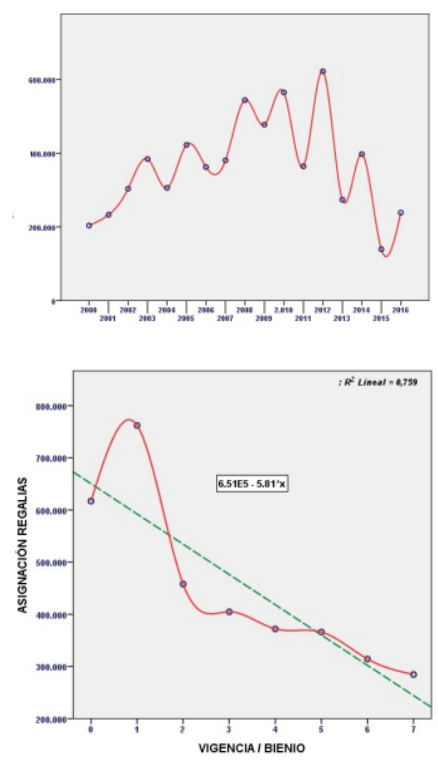

Figura 1. Ingresos por regalías y proyecciones SGR en millones de pesos departamento de Casanare
Cuando analizamos los ingresos de capital y los ingresos por regalías se observa una variación en el año 2012, esto obedece a los pagos por concepto de liquidación de regalías, lo que generó un mayor valor de giro por parte del Ministerio de Hacienda, que en el acto legislativo de creación del SGR incluyó estos pagos a los Departamentos productores. Adicionalmente se observa una variación negativa en los años 2013 y 2015 respectivamente, esto obedece a que desde el año 2013 los presupuestos por regalías son bianuales y por lo general las entidades territoriales están ejecutando sus proyectos en el segundo año y es ahí cuando se genera el desembolso de los recursos por lo tanto es en el segundo año del bienio se ve reflejado el incremento.

Teniendo un panorama poco alentador para las finanzas del departamento de Casanare, conforme se ha analizado la tendencia y dependencia de los recursos provenientes de regalías petroleras, es importante avanzar en la búsqueda de alternativas que permitan sustituir esta fuente de ingresos.
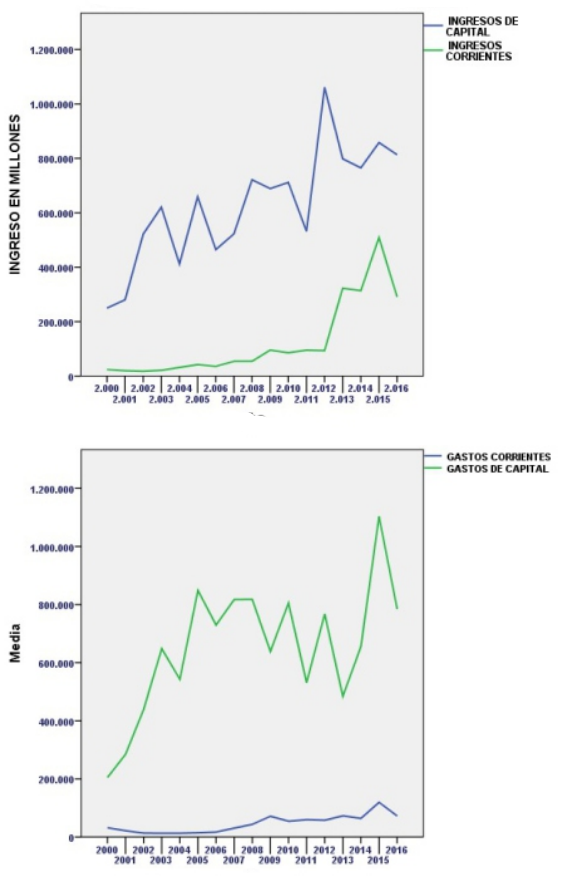

Figura 2. Ingresos de capital Vs. Ingresos corrientes Gastos corrientes Vs. Gastos de capital en millones

La composición del gasto, está influenciada en gran medida por los gastos de capital, asociados a los compromisos por concepto de regalías, como se puede observar en la anterior figura, la proporción respecto a los gastos corrientes es superior. Esto indica que el Departamento de Casanare tiene una dependencia muy alta de los recursos provenientes deISGR 
a los compromisos por concepto de regalías, como se puede observar en la anterior figura, la proporción respecto a los gastos corrientes es superior. Esto indica que el Departamento de Casanare tiene una dependencia muy alta de los recursos provenientes del SGR.

Con un modelo estadísticamente significativo y mediante la técnica de regresión lineal simple se determinó que existe una correlación entre la variable dependiente asignación de regalías y la variable independiente tiempo, la serie de tiempo presenta una correlación negativa y con un coeficiente de determinación de 0.75 , que es significativo para el modelo establecido y se prevé un decrecimiento acelerado para el departamento de Casanare en los próximos años bajo el actual SGR.
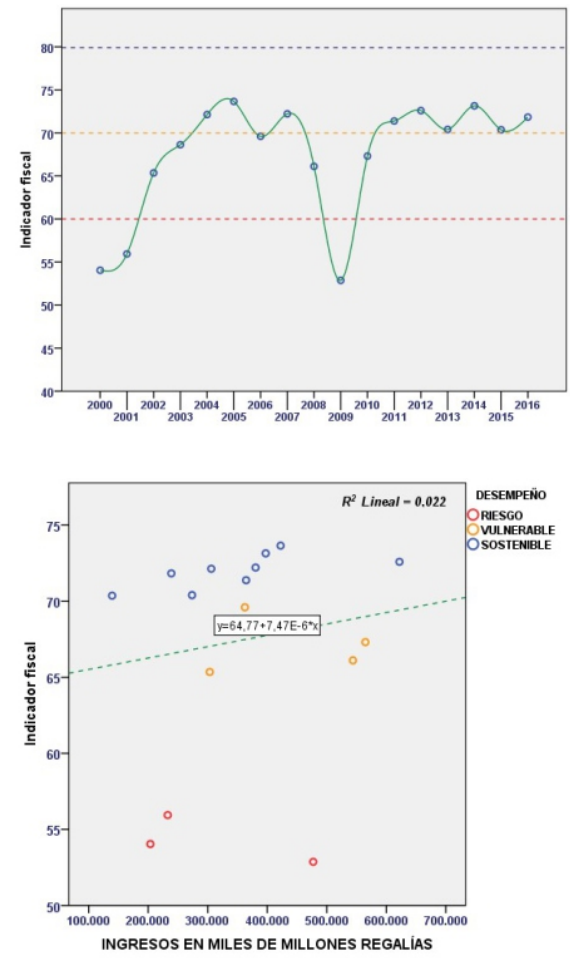

Figura 3. Indicador fiscal - Correlación entre el desempeño fiscal e ingresos de regalías

Se observa que cuando existe la dependencia por las regalías, aumenta la probabilidad de riesgo.

Cuando se estableció definir la relación entre la asignación de regalías y el desempeño fiscal del Departamento, lo primero que se quiso conocer fue la correlación entre las variables y no se encontró un valor estadísticamente significativo, teniendo un valor para el coeficiente de determinación de 0.022 , este valor indica que no existe correlación entre las variables objeto de análisis, esto quiere decir, que a partir de la entrada del SGR el departamento mejoró sus indicadores ley 617 de 2000, tal como se analizó anteriormente.

Cuando se planteó establecer el coeficiente de determinación de cada una de las variables que conforman los criterios de evaluación del desempeño fiscal del departamento con el indicador fiscal, con el propósito de establecer estadísticamente las variables que más le aportan al indicador (figura 4); Observamos lo siguiente:
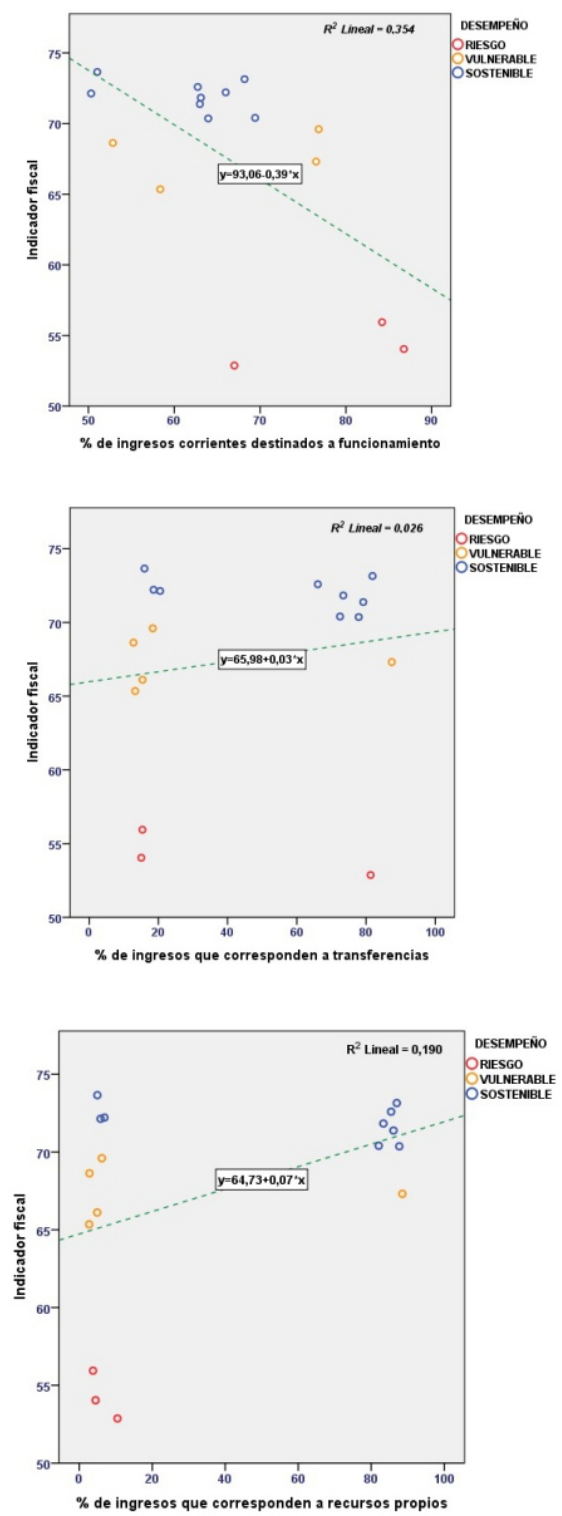

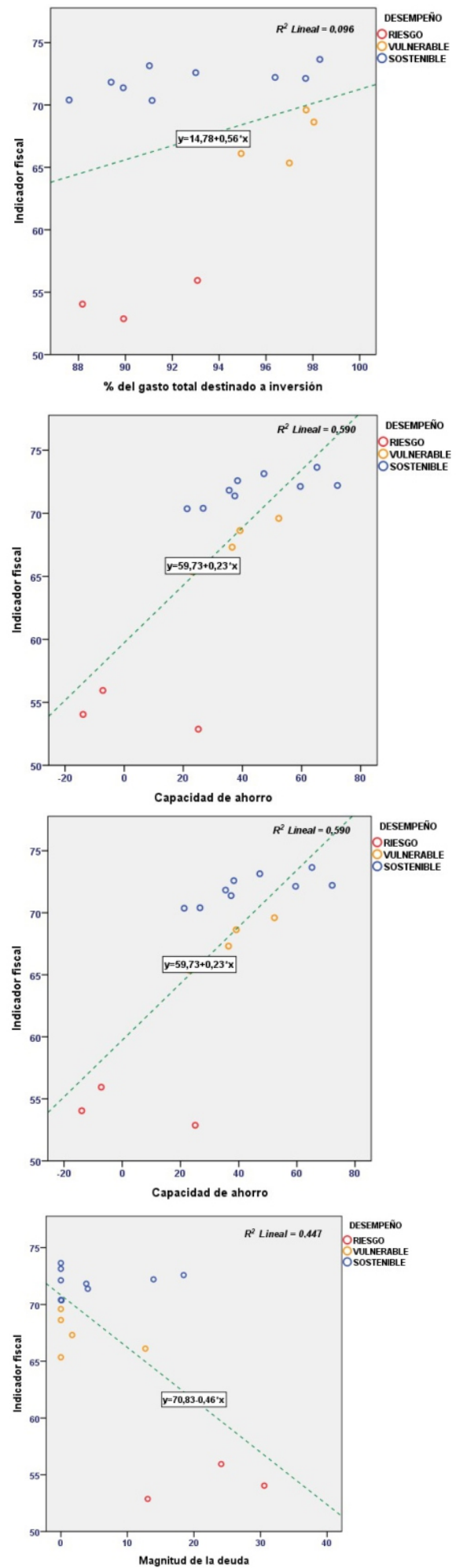

Figura 4. Correlación entre indicadores para medir desempeño fiscal del Departamento de Casanare e indicador fiscal

De lo anterior se puede inferir estadísticamente:

A mayor porcentaje de ingresos corrientes destinados a funcionamiento, mayor probabilidad de que el departamento esté en riesgo.

$\checkmark$ El porcentaje de ingresos que corresponden a transferencias es indiferente, respecto a los resultados del indicador fiscal.

$\checkmark$ El porcentaje de ingresos que corresponden a recursos propios, es indiferente respecto a los resultados del indicador fiscal.

$\checkmark$ A menor porcentaje del gasto destinado a inversión, mayor probabilidad de que el Departamento esté en riesgo.

$\checkmark$ A mayor capacidad de ahorro del ente territorial, menor probabilidad de que el Departamento esté en riesgo.

$\checkmark$ Cuanto mayor sea la magnitud de la deuda, mayor probabilidad de que el Departamento esté en riesgo

\section{Propuesta}

Se recomienda fortalecer la gestión de la construcción de nuevas capacidades productivas en el Departamento de Casanare.

Desde la perspectiva, es indispensable que exista un equilibrio entre el modelo de producción y desarrollo sostenible, toda iniciativa o proyecto productivo que se promueva con participación del Departamento, deberán involucrar los determinantes, sociales, económicos y ambientales, en especial el acatamiento a la regulación sobre limitación del uso de los recursos naturales y los lineamientos del ordenamiento territorial.

En el departamento se requiere tener un papel más directo y representativo para ayudar a la recuperación económica de la región, sin embargo se tienen algunas restricciones legales y fiscales. De un lado el Departamento tiene competencias de intermediación entre la nación y los Municipios, en las cuales no puede intervenir directamente, y de otro lado, la reducida capacidad de recursos para inversión deja un margen estrecho para direccionar proyectos hacia la reactivación económica de la Región, a través de un conjunto de estrategias que permitan actuar de manera coherente y eficaz en el corto y mediano plazo para contrarrestar esta desaceleración económica.

En el corto plazo, se debe propender por la recuperación social y económica del Departamento, con la participación de gremios, actores, sectores e instituciones que muestren su compromiso con el futuro de Casanare, con el propósito 
fundamental de concertar un plan de choque para la generación de empleo, que contribuya con la reactivación inmediata de la economía, especialmente de aquellos sectores más golpeados y con menor capacidad de adaptación.

En cuanto al mediano plazo, se debe orientar a fortalecer la vocación agropecuaria, como las apuestas más estratégicas y viables, que irán a reemplazar la economía petrolera. De igual modo, se impulsarán las apuestas productivas con posibilidades de crecimiento, que ayuden a generar nuevas opciones de emprendimiento, contribuyan a la diversificación productiva y a desarrollar el potencial agroindustrial y turístico del Departamento.

\section{CONCLUSIONES :}

En Casanare, la administración departamental debe partir de la nueva realidad y hacer un esfuerzo para tener recursos propios y usar las transferencias y las regalías, inmediatamente no tan impactadas, para construir nuevas capacidades productivas, en particular en la agricultura y la ganadería.

La coyuntura actual lleva a una reducción forzada de la dependencia del petróleo, lo cual tiene un efecto positivo en favor de un crecimiento menos desbalanceado entre los diferentes sectores de la economía.

La difícil situación por la que atraviesa la economía departamental, nos afecta a todos, pero especialmente, tiene una mayor connotación en aquellos negocios, pequeñas y medianas empresas o personas naturales que tenían vínculos contractuales con sector público Departamental.

Hay un panorama poco alentador para las finanzas del Departamento de Casanare, conforme se ha analizado la tendencia y dependencia de los recursos provenientes de regalías petroleras, es importante avanzar en la búsqueda de alternativas que permitan sustituir esta fuente de ingresos.

El Departamento va alcanzando mejores índices de desempeño fiscal, por lo menos se mantiene en un desempeño sostenible, esto significa que la entidad a partir de la reforma al sistema de regalías mejoró su desempeño fiscal.

Se mantiene la proporción de los recursos de libre destinación destinados a los gastos de operación de la administración central.
Se disminuye la deuda del Departamento considerablemente desde el año 2012, cuando la reforma del SGR, destina parte de su presupuesto para cancelarla parcial o totalmente.

La dependencia de las transferencias sigue siendo alta y con tendencia al crecimiento, resulta ser poco prudente esta dependencia y en gran medida se explica por las transferencias del SGP para educación y salud.

Aumenta el recaudo por concepto de impuestos, es decir, los ingresos corrientes o de libre destinación, tales como los ingresos tributarios.

Las variables que presentan mayor relación con el indicador fiscal son el porcentaje de ingresos corrientes destinados a funcionamiento de la administración departamental y el porcentaje del gasto toral destinado a inversión, dos variables trascendentales y que tienen una relación directa con el gasto del ente territorial.

A mayor porcentaje del gasto destinado a inversión por parte del Departamento, encontramos mejor desempeño fiscal.

A mayor porcentaje de ingresos corrientes destinados a funcionamiento mayor probabilidad de que el Departamento esté en riesgo.

El porcentaje de ingresos que corresponden a transferencias es indiferente respecto a los resultados del indicador fiscal.

El porcentaje de ingresos que corresponden a recursos propios es indiferente respecto a los resultados del indicador fiscal.

A menor porcentaje del gasto destinado a inversión mayor probabilidad de que el Departamento esté en riesgo.

A mayor capacidad de ahorro del ente territorial, menor probabilidad de que el Departamento esté en riesgo.

Cuanto mayor sea la magnitud de la deuda, mayor probabilidad de que el Departamento esté en riesgo. 


\section{BIBLIOGRAFÍA:}

ACTO LEGISLATIVO 05 DE 2011 (Secretaría Jurídica Distrital de la Alcaldía Mayor de Bogotá D.C.). Recuperado el 17 de Septiembre de 2017, de http://www.alcaldiabogota.gov.co/sisjur/

ASAMBLEA NACIONAL CONSTITUYENTE, Artículo 360 (CONSTITUCIÓN POLITICA DE COLOMBIA 1991).

Banco de la República. (2014). Composición de la Economía del Departamento de Casanare. Centro Regional de Estudios Económicos.

CAMARA DE COMERCIO DE CASANARE. (junio de 2017). Informe de Gestión 2016.

Recuperado el 06 de septiembre de 2017, de http://cccasanare.co/wpcontent/uploads/2017/06/No.-145-A-GAPPPresentaci\%C3\%B3n-EPE-Yopal-201620171.pdf

CONGRESO DE COLOMBIA. (18 de Julio de 2011). ACTO LEGISLATIVO 05 DE 2011. (Secretaría Jurídica Distrital de la Alcaldía Mayor de Bogotá D.C.) Recuperado el 06 de septiembre de 2017, de http://www.alcaldiabogota.gov.co/sisjur/norm as/Norma1.jsp?i=43391

DANE. (octubre de 2016). INFORME DE COYUNTURA ECONÓMICA REGIONA. (DANE) Recuperado el 29 de Julio de 2018, de https://www.dane.gov.co/files/icer/2015/ICE R_Casanare_2015.pdf

Departamento Nacional de Planeación. (2017). Actualización de la cartilla: "Las Regalías en Colombia". Segunda, 11.

DNP. (2018). Desempeño Fiscal. Recuperado el 27 de Julio de 2018, de Dirección de Desarrollo
Territorial Sostenible:

https://www.dnp.gov.co/programas/desarroll o-territorial/EstudiosTerritoriales/Indicadores-y-

Mediciones/Paginas/desempeno-fiscal.aspx

Repositorio Universidad del Rosario. Recuperado el 29 de Julio de 2018, de http://repository.urosario.edu.co/bitstream/ha ndle/10336/8681/102076325117.pdf?sequence $=17$

Sentencia, Sentencia C-541/11 (CORTE CONSTITUCIONAL 2011).

Sentencias, SENTENCIAS: C-800/2008 - C317/2012 (CORTE CONSTITUCIONAL 2008-2012). 